\title{
Coexistence of primary colorectal follicular lymphoma and multiple myeloma: a case report
}

This article was published in the following Dove Press journal: International Journal of General Medicine

\author{
Makoto Saito' \\ Kencho Miyashita ${ }^{2}$ \\ Yosuke Miura ${ }^{3}$ \\ Reiki Ogasawara' \\ Minoru Kanaya' \\ Koh Izumiyama' \\ Akio Mori' \\ Takeshi Kondo' \\ Masanori Tanaka' \\ Masanobu Morioka' \\ Shinya Tanaka ${ }^{4}$
}

'Department of Internal Medicine and Hematology, Aiiku Hospital, Sapporo, Japan; ${ }^{2}$ Department of Gastroenterology, Aiiku Hospital, Sapporo, Japan; ${ }^{3}$ Department of Internal Medicine, Megumino Hospital, Eniwa, Japan; ${ }^{4}$ Department of Cancer Pathology, Hokkaido University Graduate School of Medicine, Sapporo, Japan
Correspondence: Makoto Saito Department of Internal Medicine and Hematology, Aiiku Hospital, Minami 4 Nishi 25, Chuo-ku, Sapporo 064-0804, Japan

Tel +8 | | | 56322 | |

Fax +8I II 522 169|

Email ikyoku@aiiku-hp.or.jp

\begin{abstract}
Colorectal follicular lymphoma (FL) occurs less frequently than duodenal-type FL, which is an established entity, and primary multiple FL only involving the colon is rare. Furthermore, the coexistence of lymphoma and multiple myeloma (MM) within the same patient is rare and the current study reports such a case. The patient was an asymptomatic 62-year-old man. He underwent colonoscopy screening, which revealed at least five polypoid tumors from the cecum to the rectum. Biopsy samples stained positive for CD20 and B-cell lymphoma 2 (BCL2) but stained negative for $\mathrm{CD} 10$, and fluorescence in situ hybridization analysis identified $I G H / B C L 2$ in $95.2 \%$ of the tumor cells. Based on these findings, the patient was diagnosed with FL. On the bone marrow aspirate, the plasma cell count was $30 \%$ of all nucleated cells. Bence-Jones $\kappa$-type protein was detected by protein electrophoresis in serum and urine. The serum-free light chain $\kappa / \lambda$ level was significantly elevated (484.3). Thus, the patient was also diagnosed with MM. Both FL and MM were targeted therapeutically; rituximab and bendamustine were effective for FL, and lenalidomide and low-dose dexamethasone were effective for MM. The patient was treated for 3 years and 7 months and, until now, was off-treatment for 4 years without rapid progression of the two malignancies. Although both diseases are still present, the patient has maintained stable disease. Our findings suggest that lymphoma and MM should be targeted separately as independent hematological malignancies when they occur concurrently.
\end{abstract}

Keywords: follicular lymphoma, colon, multiple myeloma, coexistence, treatment

\section{Introduction}

Follicular lymphoma (FL) is the most common indolent B-cell lymphoma. FL arising in the second portion of the duodenum has some distinct clinical features different from their nodal counterpart. ${ }^{1,2}$ It was referred to as "primary intestinal FL" in the fourth edition of the WHO classification as one of the variants of $\mathrm{FL}^{3}$ and as "duodenal-type FL" in the revised fourth edition. ${ }^{4}$ Surveillance of the small bowel in patients with duodenal-type FL reveals involvement of the more distal small intestine in $80 \%-85 \%$ of cases. ${ }^{5,6}$ In contrast, colorectal involvement is considered to be less frequent in cases of FL. ${ }^{7}$ For example, Takata et $\mathrm{al}^{6}$ reported that among the 125 cases of intestinal FL, only 5 cases originated from the colon. In addition, primary multiple colorectal FL has rarely been reported. ${ }^{8,9}$

The simultaneous appearance of lymphoma and multiple myeloma (MM) is also a rare phenomenon. ${ }^{10}$ We report an extremely rare case of both primary multiple colorectal FL and MM simultaneously occurring in a single patient who was followed up for $>7$ years. 


\section{Case report}

The patient was a 62-year-old man who was asymptomatic and had no noteworthy medical history. He was referred to our hospital after his screening colonoscopy suggesting the presence of malignant lymphoma in the large intestine. The tumors were mainly found in the ascending colon (A-colon), and at least five lesions were found from the cecum to the rectum. The lesion in the A-colon had a unique form resembling a mass of small submucosal tumors (Figure 1A), whereas the other lesions were small and isolated polypoid feature. Abnormal telangiectasia was observed on the surface of these lesions (Figure 1B and C).

These tumor lesions were biopsied. Histopathology revealed small- to medium-sized lymphoid cells with mild atypia proliferation and follicular pattern (Figure 2A). Immunohistochemically, the tumor cells stained strongly positive for CD20 and B-cell lymphoma 2 (BCL2) (Figure 2B and C) but were negative for CD3, CD5, CD10, and cyclin D1. The Ki-67 labeling index was 5-10\%. Fluorescence in situ hybridization (FISH) analysis identified IGH/BCL2 in $95.2 \%$ of the tumor cells (Figure 3). Based on these results, the patient was diagnosed with FL (Grade 1).
The blood test results on admission revealed a white blood cell count of 5,800/ $\mathrm{LL}$ (normal range: 4,000-8,000/ $\mu \mathrm{L})$, a hemoglobin level of $14.2 \mathrm{~g} / \mathrm{dL}(14.0-18.0 \mathrm{~g} / \mathrm{dL})$, and a platelet count of 192,000 cells $/ \mu \mathrm{L}(120,000-400,000$ cells $/ \mu \mathrm{L})$. Lactate dehydrogenase, albumin, and $\beta 2$-microglobulin levels were $168 \mathrm{IU} / \mathrm{L}(120-245 \mathrm{IU} / \mathrm{L}), 4.5 \mathrm{~g} / \mathrm{dL}(3.8-5.3 \mathrm{~g} / \mathrm{dL})$, and $1.1 \mathrm{mg} / \mathrm{L}(<2.0 \mathrm{mg} / \mathrm{L})$, respectively. Interleukin-2 receptor was $281 \mathrm{U} / \mathrm{mL}(121-613 \mathrm{U} / \mathrm{mL})$, and all other test results were normal. Contrast computed tomography (CT) and [fluorine18]-fluorodeoxy-glucose-positron emission tomography (18F-FDG-PET)/CT revealed abnormal uptake in the A-colon, which is consistent with malignant lymphoma (Figure 4). There were no other abnormal areas. Esophagogastroduodenoscopy, video capsule endoscopy, and double balloon enteroscopy (anterograde insertion) were performed, revealing no additional tumor lesions from the esophagus to the terminal ileum. This suggested that the patient had primary multiple FL that only involved the colon (Lugano Stage I, FL International Prognostic Index [FLIPI]; FLIPI low risk, FLIPI 2 intermediate risk).

In contrast, bone marrow aspiration revealed the frequency of plasma cells to be $30 \%$ of all nucleated cells (Figure 5). IGH/BCL2 was not detected in FISH analysis.
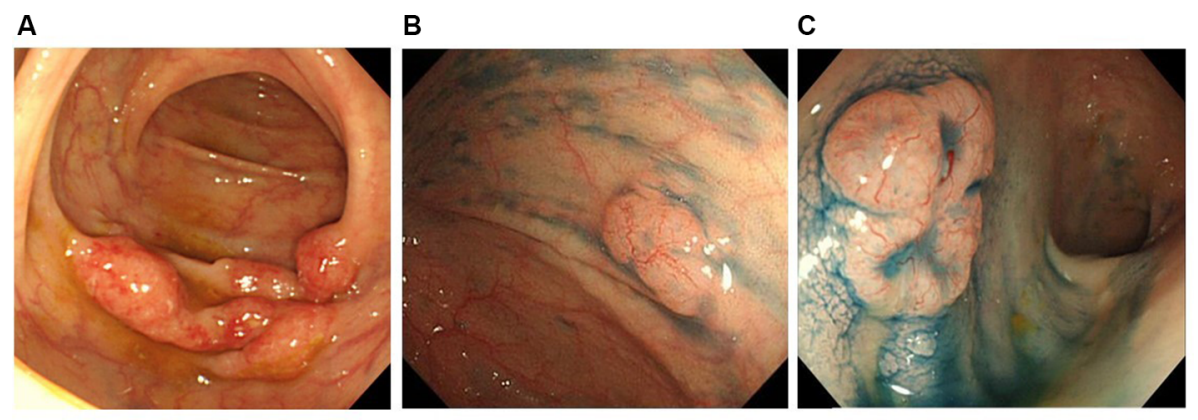

Figure I Colonoscopy findings including Indigo carmine spraying (A, ascending colon; B, cecum; C, rectum).

Notes: (A) Mass of the submucosal-like tumor. (B and $\mathbf{C})$ Isolated small polypoid tumors. An abnormal microvascular tree was noted on the surface of these lesions.

A

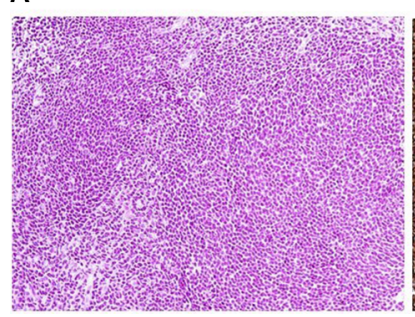

B

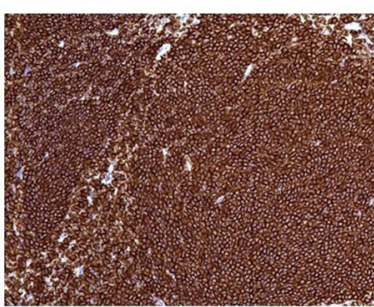

C

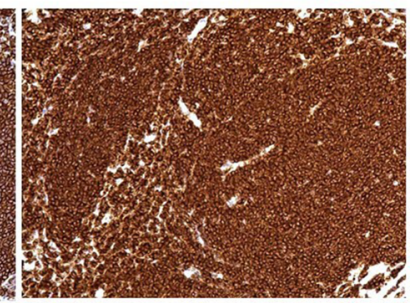

Figure 2 Histopathology findings (A, hematoxylin-eosin staining; B, CD20 immunostaining; C, BCL2 immunostaining, $\times 100)$.

Notes: (A) Small- to medium-sized lymphoid cells with mild atypia proliferation and follicular patterns were observed. (B and $\mathbf{C}$ ) The tumor cells stained strongly positive for CD20 and BCL2. 


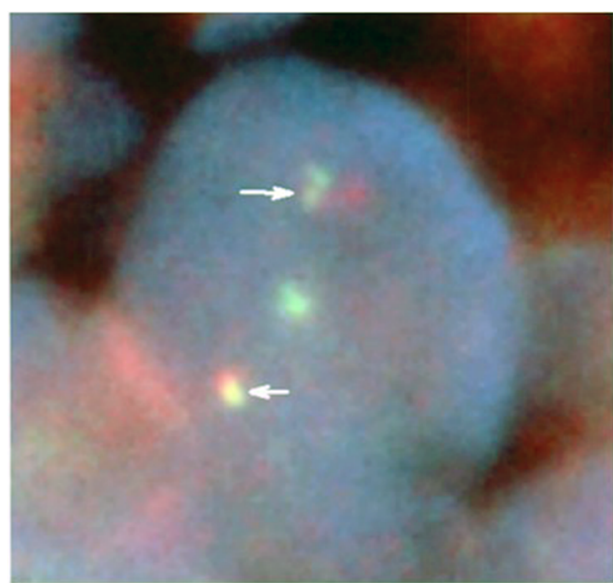

Figure $3 \mathrm{lgH} / \mathrm{BCL} 2$ rearrangement by FISH analysis.

Notes: IgH probe for $14 q 32$ (green signals) and $B C L 2$ probe for $18 q 21$ (orange signals) and arrows (yellow signals) indicate $/ g H / B C L 2$ fusion. We detected $/ g H / B C L 2$ fusion in $95.2 \%$ of tumor cells.

Abbreviation: FISH, fluorescence in situ hybridization.

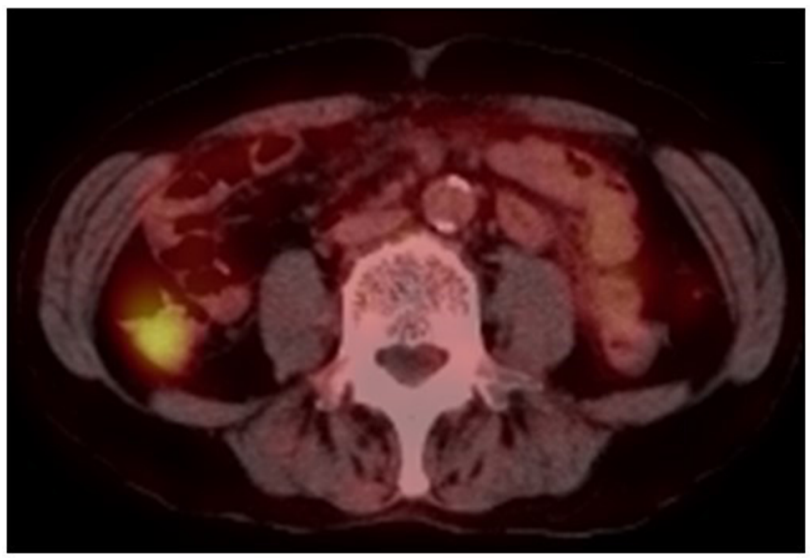

Figure 4 I8F-FDG-PET/CT finding.

Notes: In the ascending colon, abnormal uptake that was consistent with malignant lymphoma was observed. SUVmax was 4.09 .

Abbreviations: CT, computed tomography; I8F-FDG-PET, [fluorine-18]fluorodeoxy-glucose-positron emission tomography; SUVmax, maximum standardized uptake value.

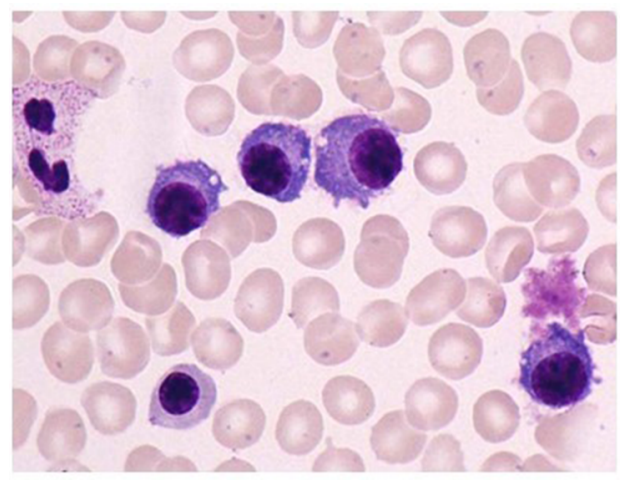

Figure 5 Bone marrow aspiration smear (May-Giemsa staining, $\times I, 000$ ). Note: The frequency of plasma cells was $30 \%$ of all nucleated cells.
Bence-Jones $\kappa$-type protein was detected by protein electrophoresis in serum and urine. In addition, the serum-free light chain (sFLC) $\kappa / \lambda$ level was significantly elevated (247.0 [2.42-18.92] mg/L/0.51 [4.44-26.18] mg/L =484.3 [0.248-1.804]). Because there was no myeloma-related organ or tissue impairment, the patient was diagnosed with smoldering MM (SMM; Bence-Jones K-type). The patient was later re-diagnosed with MM based on the revised diagnostic criteria of the International Myeloma Working Group, ${ }^{11}$ which included an sFLC ratio of $\geq 100$ as one of the myelomadefining events. The patient had the following two coexisting hematological malignancies: FL (Lugano Stage I) and MM (SMM); his performance status (PS) was 0 .

We obtained informed consent from the patient to target both FL and MM therapeutically. The treatment by chemotherapy was initiated with three cycles of rituximab (RTX) + cyclophosphamide, vincristine, prednisone $(\mathrm{COP}) / \mathrm{mel}$ phalan, prednisone (MP) ${ }^{12}$ (vincristine + cyclophosphamide [days 1, 8, and 15] + melphalan [days 29-31], + prednisolone [days 1-3, 8-10, 15-17, and 29-31]), followed by three cycles of RTX + bendamustine. RTX was then administered twice as a single agent. $\mathrm{Rd}$ (lenalidomide and low-dose dexamethasone) was initiated $\sim 9$ months after starting the treatment, and a total of 37 cycles were administered. At 3 years and 7 months after the treatment, all FL lesions, excluding the lesion in the A-colon, disappeared. The tumor lesion in the A-colon was considered to have achieved complete response (CR) based on endoscopy. However, pathological findings suggested that the lesion was suspected of minimal residual disease. The plasma cell count in bone marrow decreased to $\leq 5 \%$ and the $\kappa / \lambda$ level was in $20 \mathrm{~s}$. However, serum protein electrophoresis suggested the presence of $M$ protein. Thus, MM was considered to have achieved partial response (PR). Currently, the patient has been off-treatment for $>4$ years. Although both hematological neoplasms remain, the patient has maintained stable disease without rapid progression. Currently, he is maintaining PS0 at the age of 70 years.

Written informed consent was obtained from the patient for publication of this case report.

\section{Discussion}

On the immunophenotype of duodenal-type FL, the tumor cells are positive for CD20 and BCL2 similar to that of nodal FL. ${ }^{4}$ Reduced CD10 expression was more frequent in duodenal-type FL than in nodal FL and significantly involved the stomach or large intestine. ${ }^{13}$ For the diagnosis of FL cases with reduced CD10 expression and BCL2-positive 
follicles, molecular analysis should be performed. In this study, because a high $I G H / B C L 2$ expression (95.2\%) was noted in the tumor cells by FISH analyses, this patient was diagnosed with FL.

The characteristic endoscopic feature of duodenal-type FL is multiple small whitish polyps or whitish granules. ${ }^{1,5}$ The endoscopic features of colorectal FL have not yet been fully clarified due to its rarity. Iwamuro et al ${ }^{14}$ recently reported 12 cases of colorectal FL presenting with papular (four cases), polypoid (four cases), and flat elevated (four cases) lesions, and they found no erosion or ulcers. They also suggested that polypoid-type colorectal FL lesions may grow further, developing a submucosal tumor-like appearance. Consistent with this study, we demonstrated that five FL lesions in the colon were polypoid, and the largest lesion in the A-colon resembled a mass of submucosal tumors.

Duodenal-type FL is a slow-growing and low-grade malignancy, and patients are either monitored without therapy (watch and wait) or treated actively., ${ }^{5,6,15}$ For colorectal FL, there is no consensus on the optimal treatment option because the progression of the disease remains unclear and there have not been any large, long-term studies on clinical course. Our patient had MM as well as FL, and for such cases too, there are no standardized therapeutic management strategies. Although FL was in Stage I, we did not select radiation therapy because multiple lesions in the colon were found from the cecum to the rectum. As with treatment for nodal FL, we performed chemotherapy combining RTX + COP or RTX + bendamustine and achieved treatment response. For MM, we administered $\mathrm{Rd}$ and achieved treatment response. Consistent with our findings, early treatment with lenalidomide and dexamethasone in patients with high-risk SMM significantly delayed the time to progression to symptomatic disease and resulted in an overall survival benefit. ${ }^{16}$

The simultaneous occurrence of lymphoma and MM within the same patient is rare. ${ }^{10}$ Their clonal relationship has been controversially discussed, such as in a previous report suggesting that coexisting non-Hodgkin's lymphoma (NHL) and MM may arise from pathogenetic transformation of lymphoma cells into plasma cells. ${ }^{17}$ Hasskarl et al documented 589 patients with MM, of whom 13 patients $(2.2 \%)$ had coexisting hematological neoplasms. Acute myeloid leukemia (AML)/myelodysplastic syndrome (MDS) (six patients) and lymphoma (seven patients; chronic lymphocytic leukemia $[\mathrm{CLL}]=$ five patients, Hodgkin's lymphoma [HL] = one patient, and NHL [unclassified] = one patient) occurred with MM. Of note, in five of the six patients, AML/MDS developed after MM, whereas in six of the seven patients, lymphoma developed prior to MM or was simultaneously diagnosed with MM. The prognosis of MM patients with additional malignancies, including solid tumors, is poorer with subsequent differing neoplasms. ${ }^{10}$ Pantic et al reported that in four of the five patients with concomitant CLL and $\mathrm{MM}$, the biclonal relationship of both diseases was resolved by combined single-nucleotide polymorphism mapping array and FISH analyses on bone marrow smears. Patients with concomitant B-CLL and MM may have a less favorable MM course, exhibiting adverse prognostic features and early death due to myeloma progression. ${ }^{18} \mathrm{HL}$ and other NHL, such as mantle cell lymphoma, with MM seem to be of different cellular origins. ${ }^{19}$

To our knowledge, this is the first case report of the simultaneous occurrence of FL and MM. We have not yet elucidated the underlying mechanisms of the occurrence of FL and MM, including which arose first and how they affected each other. Because the primary organs were distinct (the colon and bone marrow), we suspect that this is an extremely rare case of nearly simultaneous presence of two independent FL and MM clones. Although the treatments did not lead to CR of FL or MM, our patient has maintained stable disease for $>7$ years without any rapid progression or occurrence of new malignancies. With the simultaneous occurrence of MM and lymphoma other than CLL, our findings suggest that these diseases should be targeted separately as independent hematological malignancies using well-established treatment options for each of them.

\section{Author contributions}

Saito $\mathrm{M}$ conceived the design of the study and prepared the manuscript. Miyashita K and Miura Y were involved in endoscopic procedure. Tanaka $\mathrm{S}$ involved in pathological procedure. All authors contributed toward data analysis, drafting and revising the paper and agree to be accountable for all aspects of the work.

\section{Disclosure}

The authors report no conflicts of interest in this work.

\section{References}

1. Yoshino T, Miyake K, Ichimura K, et al. Increased incidence of follicular lymphoma in the duodenum. Am J Surg Pathol. 2000;24(5):688-693.

2. Shia J, Teruya-Feldstein J, Pan D, et al. Primary follicular lymphoma of the gastrointestinal tract: a clinical and pathologic study of 26 cases. Am J Surg Pathol. 2002;26(2):216-224.

3. Harris NL, Swerdlow SH, Jaffe ES, Ott G. Follicular lymphoma. In: Swerdlow SH, Campo E, Harris NL, Jaffe ES, Pileri SA, Stein H, Thiele J, Vardiman JW, editors. WHO Classification of Tumours of Haematopoietic and Lymphoid Tissues. Lyon, France: IARC press; 2008:220-226. 
4. Jaffe ES, Harris NL, Swerdlow SH, Ott G, Nathwani BN. Follicular lymphoma. In: Swerdlow SH, Campo E, Harris NL, Jaffe ES, Pileri SA, Stein H, Thiele J, Arber DA, Hasserjian RP, Le Beau MM, Orazi A, Siebert R, editors. WHO Classification of Tumours of Haematopoietic and Lymphoid Tissues. Lyon, France: IARC press; 2017:266-277.

5. Schmatz AI, Streubel B, Kretschmer-Chott E, et al. Primary follicular lymphoma of the duodenum is a distinct mucosal/submucosal variant of follicular lymphoma: a retrospective study of 63 cases. J Clin Oncol. 2011;29(11):1445-1451.

6. Takata K, Okada H, Ohmiya N, et al. Primary gastrointestinal follicular lymphoma involving the duodenal second portion is a distinct entity: a multicenter, retrospective analysis in Japan. Cancer Sci. 2011;102(8):1532-1536.

7. LeBrun DP, Kamel OW, Cleary ML, Dorfman RF, Warnke RA. Follicular lymphomas of the gastrointestinal tract. Pathologic features in 31 cases and bcl-2 oncogenic protein expression. Am J Pathol. 1992;140(6):1327-1335.

8. Hiraide T, Shoji T, Higashi Y, Matsuda I, Terada T. Extranodal multiple polypoid follicular lymphoma of the sigmoid colon. Gastrointest Endosc. 2011;73(1):182-184.

9. Ferreira A, Gonçalves R, Rolanda C. A different kind of colon polyp. Gastroenterology. 2012;143:1693-1694.

10. Hasskarl J, Ihorst G, De Pasquale D, et al. Association of multiple myeloma with different neoplasms: systematic analysis in consecutive patients with myeloma. Leuk Lymphoma. 2011;52(2):247-259.

11. Rajkumar SV, Dimopoulos MA, Palumbo A, et al. International myeloma working group updated criteria for the diagnosis of multiple myeloma. Lancet Oncol. 2014;15:e538-e548.
12. Takenaka T, Shirakawa S, Mikuni C, et al. Alternating combination chemotherapy COP (cyclophosphamide, vincristine, prednisone) and MP (melphalan, prednisone) in multiple myeloma: a multicenter phase II study (JCOG8906). Lymphoma study group of the Japan clinical oncology group. Jpn J Clin Oncol. 1999;29(10):485-489.

13. Ohnishi N, Takata K, Miyata-Takata T, et al. CD10 down expression in follicular lymphoma correlates with gastrointestinal lesion involving the stomach and large intestine. Cancer Sci. 2016;107(11): 1687-1695.

14. Iwamuro M, Okada $\mathrm{H}$, Takata $\mathrm{K}$, et al. Colorectal manifestation of follicular lymphoma. Intern Med. 2016;55(1):1-8.

15. Mori M, Kobayashi Y, Maeshima AM, et al. The indolent course and high incidence of $\mathrm{t}(14 ; 18)$ in primary duodenal follicular lymphoma. Ann Oncol. 2010;21(7):1500-1505.

16. Mateos MV, Hernández MT, Giraldo P, et al. Lenalidomide plus dexamethasone for high-risk smoldering multiple myeloma. $N$ Engl J Med. 2013;369:438-447.

17. Lee GC, Hong JS, Lee $\mathrm{KH}$, et al. A case of coincident multiple myeloma and non-Hodgkin's lymphoma. Korean J Intern Med. 1994;9(2):113-115.

18. Pantic M, Schroettner P, Pfeifer D, et al. Biclonal origin prevails in concomitant chronic lymphocytic leukemia and multiple myeloma. Leukemia. 2010;24(4):885-890.

19. Yamaguchi M, Ohno T, Miyata E, et al. Analysis of clonal relationship using single-cell polymerase chain reaction in a patient with concomitant mantle cell lymphoma and multiple myeloma. Int J Hematol. 2001;73:383-385.
International Journal of General Medicine

\section{Publish your work in this journal}

The International Journal of General Medicine is an international, peer-reviewed open-access journal that focuses on general and internal medicine, pathogenesis, epidemiology, diagnosis, monitoring and treatment protocols. The journal is characterized by the rapid reporting of reviews, original research and clinical studies across all disease areas.

\section{Dovepress}

The manuscript management system is completely online and includes a very quick and fair peer-review system, which is all easy to use. Visit http://www.dovepress.com/testimonials.php to read real quotes from published authors. 